Mar. Drugs 2007, 5, 23-30

Marine Drugs

ISSN 1660-3397

(C) 2007 by MDPI

www.mdpi.org/marinedrugs

Full Original Paper

\title{
Large-Scale Biotechnological Production of the Antileukemic Marine Natural Product Sorbicillactone A
}

Gerhard Bringmann ${ }^{1, *}$, Tobias A. M. Gulder ${ }^{1}$, Gerhard Lang ${ }^{1,2}$, Stefanie Schmitt ${ }^{1}$, Rüdiger Stöhr $^{2}$, Jutta Wiese ${ }^{2}$, Kerstin Nagel ${ }^{2}$ and Johannes F. Imhoff ${ }^{2, *}$

${ }^{1}$ Institut für Organische Chemie, Universität Würzburg, Am Hubland, D-97074 Würzburg, Germany

${ }^{2}$ Marine Mikrobiologie, Leibniz-Institut für Meereswissenschaften, IFM-GEOMAR, Düsternbrooker Weg 20, D-24105 Kiel, Germany

* Authors to whom correspondence should be addressed; G.B. Tel. +49-931-8885323, Fax +49-9318884755, E-mail: bringman@chemie.uni-wuerzburg.de; J.F.I. Tel. +49-431-6004450, Fax +49-4316004452, E-mail: jimhoff@ifm-geomar.de

Dedicated to Prof. Dr. W.E.G. Müller on the occasion of his $65^{\text {th }}$ birthday

Received: 29 May 2007 / Accepted: 21 June 2007 / Published: 25 June 2007

\begin{abstract}
In the search for novel bioactive compounds from sponge-derived microorganisms, we have recently identified two structurally and biosynthetically unprecedented fungal metabolites, the novel-type alkaloids sorbicillactone $\mathrm{A}$ and sorbicillactone B. Sorbicillactone A is active against leukemia cells without showing notable cytotoxicity. Therefore, we have developed an efficient process for its biotechnological production and isolation on a large scale supplying sufficient material for the ongoing preclinical investigations and structure-activity relationship (SAR) studies.
\end{abstract}

Keywords: sorbicillactone A, anti-leukemic activity, large-scale production, BIOTECmarin.

\section{Introduction}

In the course of a research program ("Molecular Biotechnology and Natural Products from Marine Sponges and Sponge-associated Microorganisms”) aiming at the discovery and characterization of novel natural products, we have identified the first members of a new class of secondary metabolites, 
the sorbicillin-derived alkaloids sorbicillactone A (1) and its 2',3'-dihydroanalog sorbicillactone B (2), from a salt-water culture of a Penicillium chrysogenum strain isolated from a specimen of the Mediterranean sponge Ircinia fasciculata [1,2]. These compounds originate via a likewise unprecedented biosynthetic pathway elucidated in our laboratories by applying feeding experiments with ${ }^{13} \mathrm{C}$-labelled precursors [2].

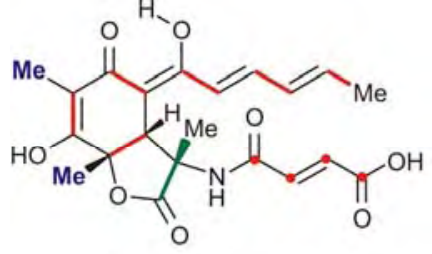

Sorbicillactone A (1)

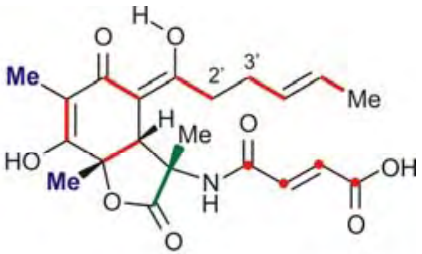

Sorbicillactone B (2)

Figure 1. Structures of sorbicillactones A (1) and B (2); the biosynthetic origins of the different molecular portions are shown in red (acetate), green (alanine), and blue ( $S$-adenosylmethionine).

Moreover, sorbicillactone A (1) exhibits a highly selective activity against the murine leukemic lymphoblast cell line L5178y, and has antiviral and neuroprotective properties [2,3]. Despite its almost identical molecular structure, $\mathbf{2}$ is significantly less active than $\mathbf{1}$ (by a factor of 10) [2,3]. Thus, for the complete evaluation of the therapeutical potential of $\mathbf{1}$, for preclinical investigations, and for SAR studies on semisynthetic analogs, a method for the large-scale production and isolation of the more promising derivative $\mathbf{1}$ was required, capable of eliminating the chromatographically almost identical analog 2 [4].

\section{Results and Discussion}

Biotechnological Production of Sorbicillactone A (1)

Sorbicillactone A (1) was first detected in a Penicillium chrysogenum isolate from the marine sponge Ircinia fasciculata (Porifera). The initial amounts produced were approximately $4 \mathrm{mg} \mathrm{l}^{-1}$ (range 2-6 $\mathrm{mg} \mathrm{l}^{-1}$ ) culture broth in surface liquid cultures. In order to increase the yield and to adapt the production to amounts sufficient for preclinical and clinical studies, growth media and culture conditions were varied. In parallel, screening experiments were performed to search for new strains with higher yields of $\mathbf{1}$.

Growth experiments with surface cultures were performed in 300-ml Erlenmeyer flasks containing $100 \mathrm{ml}$ of medium while large-scale production was done with $700 \mathrm{ml}$ medium in 2-l flasks. Cultures were inoculated with spore suspensions of Penicillium chrysogenum and incubated without shaking. The concentrations of $\mathbf{1}$ were measured by UV absorbance at $380 \mathrm{~nm}$ after separation by HPLC. Sorbicillactone A (1) was released to the culture medium but also accumulated in the mycelium of Penicillium chrysogenum. The mycelium was separated mechanically, by filtration, and $\mathbf{1}$ was extracted from the culture medium using XAD16. 


\section{Media Composition and Growth Conditions}

First experiments on the production of sorbicillactone A (1) were performed with Penicillium chrysogenum strain E01-10/3 in Wickerham medium (3.0 g yeast extract, $3.0 \mathrm{~g}$ malt extract, $5.0 \mathrm{~g}$ Bacto-peptone, and 10.0 g glucose in $1000 \mathrm{ml}$ of sea water at $30 \mathrm{PSU}$ and $\mathrm{pH} 7.3$ [5]. The initial pH of the culture media had a significant influence on the production yield. Under acidic conditions ( $\mathrm{pH} 4.0$ ) production rates were very low (15\% of maximum amounts) increasing to maximum values at $\mathrm{pH}$ 7.0. Temperature and salinity had interdependent influence on the production of 1 with optima at $28-30^{\circ} \mathrm{C}$ and $0.5-1.0 \% \mathrm{NaCl}$. $\mathrm{NaCl}$ exhibited a stronger effect and revealed higher production yields of $\mathbf{1}$ as compared to equivalent amounts of sea salts or marine water media.

\section{Production of the First 100 Grams of Substance}

In order to supply sufficient amounts of $\mathbf{1}$ for experiments on biochemical mechanisms, bioactivities, and toxicological studies, production was performed with strain E01-10/3 in modified Wickerham medium (Bacto-peptone $5.0 \mathrm{~g}$, malt extract $3.0 \mathrm{~g}$, yeast extract $3.0 \mathrm{~g}$, glucose $10.0 \mathrm{~g}$, and $0.5 \% \mathrm{NaCl}, \mathrm{pH}$ 6.6), resulting in yields of $25 \mathrm{mg} \mathrm{l}^{-1}$ culture broth on the average (ranges from 10-80 $\mathrm{mg} \mathrm{l}^{-1}$ ) after 14 days of incubation at $30^{\circ} \mathrm{C}$ in the dark.

\section{Improvement of the Culture Conditions}

Variations in the composition of complex nutrient components and single defined carbon substrates had measurable effects on the production of $\mathbf{1}$ by strain E01-10/3. Replacement of glucose in the basic Wickerham medium by other carbon sources (lactose, maltose, sucrose, fructose, galactose, trehalose, glycerol, pyruvate, acetate, or tri-sodium citrate) revealed different effects. Yields with all of these substrates were lower compared to those obtained with glucose. The highest yields were obtained with sucrose, galactose, maltose, and trehalose (approximately 60-66\% compared to glucose). With lactose as a defined carbon source, the yield was reduced to less than $50 \%$ (as compared to the yield obtained with equal amounts of glucose) and was even lower with the other carbon sources.

\section{Preparation of Spore Suspension for Inoculation}

With some of the Penicillium chrysogenum strains experiments were performed to investigate the influence of the preparation method for spore suspensions on the production of $\mathbf{1}$ using spore suspension type 2 and type 3 for strains KIPB10, KIPB11, KIPB25, H269 and L122 and in addition type 1 for strain E01-10/3. It was found that the number of spores in the inoculum and the type of spore suspension had a significant influence on the formation of the surface mycelium and on the production of $\mathbf{1}$ (see Table 1 ).

Type 1 spore suspensions were used for the mass cultivation in 2-l Erlenmeyer flasks. Spores were taken from agar plates, suspended in sterile sea water/glycerol $1 / 1(\mathrm{v} / \mathrm{v})$, and stored until use at $-20^{\circ} \mathrm{C}$. As inoculum, $100 \mu \mathrm{l}$ of this suspension were used per 700 -ml culture.

Type 2 spore suspensions were taken from 2-week old agar cultures, suspended in $0.9 \%$ aqueous $\mathrm{NaCl}$ and directly used for inoculation. 
For type 3 spore suspensions, spores were taken from 2-week old agar cultures and suspended in $0.9 \%$ aqueous $\mathrm{NaCl}$ with $0.1 \%(\mathrm{w} / \mathrm{v})$ Tween 80 immediately before inoculation into liquid cultures.

Table 1. Sorbicillactone A production by strains of Penicillium chrysogenum isolated from various marine sources; * these isolates were kindly provided by Dr. K. Schaumann (AWI, Bremen) and now are included in the collection of marine fungi of the "Zentrum für Marine Wirkstoffe” in Kiel.

\begin{tabular}{|c|c|c|c|}
\hline Strain & Source & $\begin{array}{c}\text { Quantity of } \mathbf{1} \\
\text { produced } \\
{\left[\mathrm{mg} \mathrm{l}^{-1}\right]}\end{array}$ & Conditions of cultivation \\
\hline E01-10/3* & Ircinia spec., Elba & 25 & $\begin{array}{l}\text { Modified Wickerham medium (0.5\% NaCl, pH 6.6) } \\
\text { (inoculated with spore suspension type } 1 \text { ) }\end{array}$ \\
\hline E01-10/3* & Ircinia spec., Elba & 102 & \\
\hline KIPB25 & Tubulipora spec., Baltic Sea & 99 & \\
\hline KIPB11 & Tubulipora spec., Baltic Sea & 97 & \\
\hline KIPB10 & Tubulipora spec., Baltic Sea & 96 & \\
\hline E99-2/174* & Unidentified sponge, Elba & 77 & \\
\hline $\mathrm{E} 00-7 / 22 *$ & Hamigera hamigera, Elba & 58 & \\
\hline $\mathrm{E} 01-12 / 1^{*}$ & Ircinia spec., Elba & 43 & \\
\hline R02-S2 & marine sediment, Rovinj & 26 & Modified Wickerham medium, 0.5\% NaCl, pH 7.0 \\
\hline R02-S8 & marine sediment, Rovinj & 9 & (inoculated with spore suspension type 2) \\
\hline H269 & Halichondria panicea, Baltic Sea & 9 & \\
\hline $\mathrm{R} 03-9 / 5$ & Cacospongia scalaris, Rovinj & 6 & \\
\hline H259 & Halichondria panicea, Baltic Sea & 5 & \\
\hline L122 & Laminaria saccharina, Baltic Sea & 5 & \\
\hline $\mathrm{R} 02-13 / 2$ & Acanthella spec., Rovinj & $<1$ & \\
\hline $\mathrm{R} 03-8 / 4$ & Tethya aurantium, Rovinj & $<1$ & \\
\hline H236 & Halichondria panicea, Baltic Sea & $<1$ & \\
\hline KIPB33 & Callopora aurita, Baltic Sea & 295 & \\
\hline E01-10/3* & Ircinia spec., Elba & 157 & \\
\hline KIPB25 & Tubulipora spec., Baltic Sea & 178 & \\
\hline KIPB11 & Tubulipora spec., Baltic Sea & 192 & WSP medium (inoculated with spore \\
\hline KIPB10 & Tubulipora spec., Baltic Sea & 111 & suspension type 3) \\
\hline H269 & Halichondria panicea, Baltic Sea & 11 & \\
\hline L122 & Laminaria saccharina, Baltic Sea & 17 & \\
\hline КIPB33 & Callopora aurita, Baltic Sea & 505 & $\begin{array}{l}\text { Modified WSP medium ( } 2.5 \mathrm{~g} \text { glucose and } 7.5 \mathrm{~g} \\
\text { lactose) } \\
\text { (inoculated with spore suspension type } 3 \text { ) }\end{array}$ \\
\hline
\end{tabular}




\section{Screening for New Sorbicillactone A Producers}

During isolation procedures from various marine sources a number of additional strains of Penicillium chrysogenum (identified by morphological properties of colony, cells, and sporangia, and by $18 \mathrm{~S}$ rDNA sequence comparison) were isolated. Some showed negligible production of $\mathbf{1}$, others provided highly improved production yields (see Table 1 ). New isolates of $P$. chrysogenum were obtained from marine sponges, bryozoans, algae, and also marine sediments from Baltic Sea and Mediterranean Sea habitats. The highest production yields of $\mathbf{1}$ were obtained with strains derived from bryozoans (KIPB10, KIPB11, KIPB25, and KIPB33). These phenotypic findings might indicate strain specific genetic differences, which result in various yields of $\mathbf{1}$.

\section{Selection and Improvement of Production of Sorbicillactone A (1) by Strain KIPB33}

By far the best yields were obtained with strain KIPB33 isolated from the bryozoan Callopora aurita from the Baltic Sea. If grown on WSP medium (modified Wickerham medium in which Bactopeptone was replaced by soybean peptone) yields were approximately two times higher as compared to strain E01-10/3 grown under identical conditions (100 $\mathrm{ml}$ in a $300-\mathrm{ml}$ Erlenmeyer flask at $30^{\circ} \mathrm{C}$ ). With this strain, experiments were performed in which glucose was replaced only partly by other carbon sources such that different ratios of glucose and the alternative carbon source were achieved in WSP medium. Further significant increases in yields of 1 were obtained with carbon mix B (5.0 g l ${ }^{-1}$ glucose and $5.0 \mathrm{~g} \mathrm{l}^{-1}$ lactose in modified WSP medium, $425 \mathrm{mg} \mathrm{l}^{-1}$ of $\mathbf{1}$ ) and with carbon mix C (2.5 g $\mathrm{l}^{-1}$ glucose and $7.5 \mathrm{~g} \mathrm{l}^{-1}$ lactose in modified WSP medium, $505 \mathrm{mg} \mathrm{l}^{-1}$ of $\mathbf{1}$ ).

\section{Extraction of Sorbicillactone A (1) from the Culture Media}

After separation of the mycelia and filtration of the culture broth, $\mathbf{1}$ and other secondary metabolites were bound to XAD added directly to the culture broth (10 g XAD16 $\mathrm{l}^{-1}$ ) and eluted from the XAD with methanol. The methanol extracts were concentrated in a rotary evaporator and then used for further purification.

\section{Extraction of Sorbicillactone A (1) from the XAD Eluate}

For a first crude removal of large quantities of undesired major secondary metabolites of $P$. chrysogenum, such as, e.g., meleagrin, but also other water-soluble compounds like sugars and salts, a simple distribution of the XAD extract between water $(\mathrm{pH}=7.0)$ and ethyl acetate proved highly efficient. After acidification of the remaining aqueous phase using phosphoric acid $(\mathrm{pH}=2.0)$, compound 1 was quantitatively transferred into the organic phase (ethyl acetate) by renewed extraction. The extract thus obtained already contained ca. 50\% (w/w) of sorbicillactone A (1).

\section{Purification of the Crude Extract by Fast Centrifugal Partition Chromatography (FCPC)}

To further purify the crude extract, the method of choice was FCPC [6] as a modern liquid-liquid chromatography, which, like High Speed Countercurrent Chromatography (HSCCC) [7], uses a 
2-phase solvent system for separation. The ratio of the concentrations (K value) of a compound in the stationary and the mobile phase should be in the range of 0.7 to 4.5 to allow sufficient separation within acceptable retention times. To determine suitable solvent mixtures, 1 was distributed in 2-phase systems of different compositions, and the partitioning of the compound was determined using analytical HPLC. The best solvent mixture, with a K value of 1.04, consisted of $n$-heptane / ethyl acetate $(42 \% / 58 \%)$ as the stationary phase and methanol / water (42\%/58\%) as the mobile phase, both buffered with $0.1 \%$ phosphoric acid. When using a $200-\mathrm{ml}$ rotor at a constant flow of $7 \mathrm{ml} \mathrm{min}^{-1}$ at $1200 \mathrm{rpm}$, the purification of $1.5 \mathrm{~g}$ of extract per run was possible, each run needing approximately 90 min including flushing and equilibration of the chromatographic system [8]. After evaporation of the organic solvents of the fractions containing sorbicillactone A (1), the remaining aqueous solution was exhaustively extracted with ethyl acetate. The resulting extracts almost exclusively contained $\mathbf{1}$ and $\mathbf{2}$ in their natural ratio as obtained after fermentation, i.e., with up to ca. $70 \%$ (w/w) sorbicillactone A (1).

\section{Resolution of 1 and 2 by Gel Chromatography on Sephadex LH20}

The most demanding step during the isolation process of sorbicillactone A (1) was its separation from the structurally - and thus chromatographically - almost identical 2',3'-dihydroanalog, sorbicillactone B (2). The resolution succeeded by gel chromatography on Sephadex LH-20 [9], albeit only when using a long chromatographic system (6-m column, $3.5 \mathrm{~cm}$ inner diameter) and pure methanol as the eluent. The solvent was pumped through the column by using an MPLC pump at a constant flow rate of $10 \mathrm{ml} \mathrm{min}{ }^{-1}$. For a single run $1.5 \mathrm{~g}$ of the extracts derived from the FCPC separation were dissolved in $7 \mathrm{ml}$ of methanol and loaded over an 8-ml injection loop. Sorbicillactone B (2) was eluted after ca. 7 h, directly followed by sorbicillactone A (1). Although the two compounds were not baseline separated, ca. 70\% of $\mathbf{1}$ submitted to the gel chromatography was recovered in pure fractions after the column. The remaining 30\% of 1 were still contaminated with 2 , but were easily further purified by renewed chromatography.

\section{Analysis of the Purity of Sorbicillactone A (1) Containing Fractions}

For a fast analysis of the purity of the fractions obtained after the chromatographic steps, a variety of HPLC methods were tested. Initially, the quality of the fractions was checked by using a Waters Symmetry C18 column (2.1 x $150 \mathrm{~mm}, 0.4 \mathrm{ml} \mathrm{min}^{-1}$ flow) with an isocratic solvent system (water / acetonitrile / TFA = 70/30/0.05; elution time for 1: $12.5 \mathrm{~min}$, and for 2: $13.2 \mathrm{~min}$ ). These analyses required 23 min per run (including flushing and conditioning of the column), thus consuming too much time for the analysis of a large number of fractions. The time per sample was significantly reduced to $5 \mathrm{~min}$ by utilizing a Merck Chromolith column $(4.6$ x $100 \mathrm{~mm})$ using the same solvent system as described above, but at a flow rate of $5 \mathrm{ml} \mathrm{min}^{-1}$.

To further reduce the analysis time needed per fraction of the final gel chromatography and to avoid the use of HPLC systems for these experiments, an additional method for the validation of the purity of these samples was developed, by taking advantage of the different UV properties of $\mathbf{1}$ and 2, which both significantly absorb at $300 \mathrm{~nm}$ (Fig. 2, blue bars, corresponding to the overall 
concentrations of 1 plus 2), but only 1 is UV active at $430 \mathrm{~nm}$ (Fig. 2, green bars, proportional to the concentration of $\mathbf{1}$, only). Thus, when showing a constant ratio between the measurement of the UV absorption at $300 \mathrm{~nm}$ and at $430 \mathrm{~nm}$ of a certain fraction (Fig. 2, red bars, from fractions 9 to 17), the sample exclusively contained the more slowly eluting pure sorbicillactone A (1). By applying this method, the time required for analysis of the resolved fractions was drastically reduced to below 1 minute.

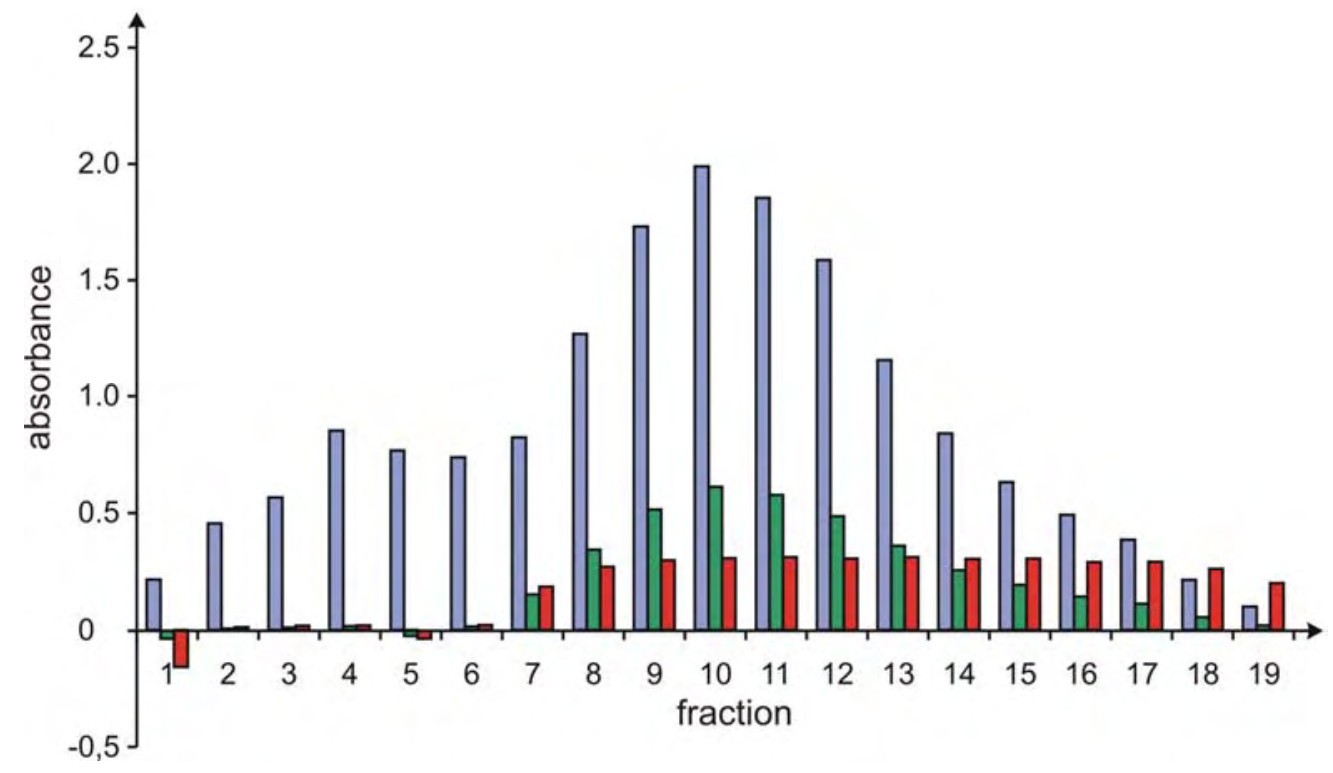

Figure 2. Rapid analysis of the sorbicillactone A/B ratio by UV measurements. The blue and green bars show the relative absorbance of the fractions at $300 \mathrm{~nm}$ and $430 \mathrm{~nm}$, respectively, while the red bars correspond to the ratios of the two absorbances.

\section{Conclusions}

A method for the biotechnological production of large quantities of the antileukemic marine compound sorbicillactone A (1) was developed. By this procedure, $100 \mathrm{~g}$ of pure sorbicillactone A (1) have been isolated for ongoing preclinical studies on this potential antitumoral drug, despite the demanding separation problem due to the co-occurrence of sorbicillactone B (2). Although significant production of $\mathbf{1}$ by Penicillium chrysogenum only succeeded in static surface cultures of the fungus, the dramatically increased yield by strain selection and the systematic variation of culture conditions now permits biotechnological production of kilogram quantities of $\mathbf{1}$, sufficient for clinical trials.

\section{Acknowledgements}

This work was supported by the project BIOTECmarin fundet by the German Bundesministerium für Bildung, Wissenschaft und Forschung (BMBF, grant nos.03F0414B and 03F0345E), the states of Schleswig-Holstein and Bayern, the University of Würzburg, by the project Zentrum für Marine Wirkstoffe funded by the Land Schleswig-Holstein, and by the Fonds der Chemischen Industrie. We thank Michaela Schraut, Stefanie Böhm, and Marion Fetters for technical assistance during the largescale isolation and Dr. R. Schmaljohann for identification of the fungi. Furthermore, we thank late 
Dr. K. Schaumann for generously providing the first sorbicillactone A producing Penicillium chrysogenum strain.

\section{References and Notes}

1. Bringmann, G.; Lang, G.; Mühlbacher, J.; Schaumann, K.; Steffens, S.; Rytik, P.G.; Hentschel, U.; Morschhäuser, J.; Brun, R. In Sponges (Porifera), Müller, W.E.G., Ed.; Springer: Berlin, 2003; Vol. 1, pp 231-253.

2. Bringmann, G.; Lang, G.; Gulder, T.A.M., Hideyuki, H.; Mühlbacher, J.; Maksimenka, K.; Steffens, S.; Schaumann, K.; Stöhr, R.; Wiese, J.; Imhoff, J.F.; Perović-Ottstadt, S.; Boreiko, O.; Müller, W.E.G. The first sorbicillinoid alkaloids, the antileukemic sorbicillactones A and B, from a sponge derived Penicillium chrysogenum strain. Tetrahedron 2005, 61, 7252-7265.

3. Bringmann, G.; Lang, G.; Mühlbacher, J.; Schaumann, K.; Steffens, S.; Müller, W.E.G. Sorbicillactone A derivatives for the treatment of tumor and viral diseases. International Patent Application WO 2004/026854 (1.4.2004).

4. Bringmann, G.; Lang, G.; Gulder, T.A.M.; Schaumann, K.; Müller, W.E.G.; Perović-Ottstadt, S.; Stöhr, R.; Wiese, J.; Schmaljohan, R.; Imhoff, J.F. Method for producing sorbicillactone A. International Patent Application WO 2005/072711 (11.8.2005).

5. Wickerham, L. J. Taxonomy of yeasts. US Dept. Technol. Bull. 1951, 1029, 1-56.

6. For more information on FCPC from KROMATON TECHNOLOGIES, see www.kromaton.com

7. Ito, Y. High Speed Countercurrent Chromatography. Crit. Rev. Anal. Chem. 1986, 17, 65-143.

8. The amount of extract purified per run was easily scaled up in a linear manner by using a 1-l rotor in the same FCPC system.

9. Henke, H. Präparative Gelchromatographie an Sephadex LH-20. Obernburg, 1994.

Samples Availability: Available from the authors.

(C) 2007 by MDPI (http://www.mdpi.org). Reproduction is permitted for noncommercial purposes. 